\title{
BLIND SOFT-DETECTION ASSISTED FREQUENCY-HOPPING MULTICARRIER DS-CDMA SYSTEMS
}

\author{
Lie-Liang Yang and Lajos Hanzo \\ Dept. of ECS, University of Southampton, SO17 1BJ, UK. \\ Tel: +44-703-593 125, Fax: +44-703-594 508 \\ Email: lh@ecs.soton.ac.uk, http://www-mobile.ecs.soton.ac.uk
}

\begin{abstract}
A slow frequency hopping, multicarrier direct-sequence, code-division multiple-access (SFH/MC DSCDMA) scheme is proposed and investigated, which has the potential of providing a joint framework for 2nd-generation narrow-band CDMA, 3rd-generation wideband CDMA and high-rate broadband access networks. Blind soft-detection of the SFH/MC DSCDMA signals is investigated, assuming that the receiver has no knowledge of the associated frequencyhopping (FH) patterns invoked. The system's performance is evaluated over the range of Nakagami multipath fading channels. The results show that the blind soft-detection not only achieves the required bit error rate performance, but it is also capable of blindly acquiring the FH patterns employed. This assists the mobile in accessing the network without the knowledge of the FH patterns or during soft hand-over.
\end{abstract}

\section{INTRODUCTION}

With the emergence of broadband wireless access networks (BRAN) requiring bandwidths substantially higher than that of the 3rd generation systems [1] it is of increasing significance to create a BRAN framework, which is backwards compatible with 2 nd and 3 rd generation schemes. An attractive candidate scheme capable of achieving these requirements is constituted by the proposed frequency-hopping (FH) based multicarrier DS-CDMA (FH/MC DS-CDMA) arrangement, where no rigid spectral segmentation is required for the co-existence of various wireless systems. Specifically, the entire bandwidth can be divided into a number of sub-bands and each sub-band can be assigned a subcarrier. A subcarrier could deliver a narrow-band IS-95 type service or - similarly to the emerging multi-carrier assisted cdma2000 system - it could invoke a number of carriers, while employing a variety of different spreading factors.

$\mathrm{FH}$ can be invoked for each user, in order to occupy the whole system's bandwidth and to efficiently utilize the system's frequency resources. Various slow and fast $\mathrm{FH}$ schemes or adaptive $\mathrm{FH}$ techniques can be supported, depending on the state-of-the-art. In FH/MC DS-CDMA sys-

The financial support of the following organisations is gratefully acknowledged: EPSRC, UK, the Comission of the European Communities, Brussels, Belgium and Motorola ECID, Swindon, UK and the Virtual Centre of Excellence (VCE) in Mobile Communications. tems the sub-bands are not required to be of equal bandwidth. Hence existing 2nd- and 3rd- generation CDMA systems can be supported using one or more subcarriers, consequently simplifying the frequency resource management and efficiently utilizing the entire bandwidth available. Furthermore, the proposed system concept lends itself to unlicensed operation, erradicating the inefficient spectral segmentation of different co-existing systems.

In this treatise we propose a FH/MC DS-CDMA system employing slow $\mathrm{FH}$ and binary modulation. The system operates in a multipath fading environment, and a maximum ratio combining (MRC) assisted RAKE receiver is used. The system's performance is investigated over various Nakagami- $m$ multipath fading channels, describing a variety of probability density functions (pdf) ranging from a Rayleigh fading channel to a non-fading Gaussian channel by varying a single parameter, namely $m$, from one to infinity [2]. We assume that the mobile station (MS) has no knowledge of the frequency-hopping $(\mathrm{FH})$ patterns employed, since it is about to attempt its initial access to the system. Hence the MS has to infer the FH pattern employed by the base station (BS) blindly. We will show that blind soft-detection using maximum likelihood detection (MLD) which is considered to be the optimum scheme - can be utilized, in order to simultaneously accomplish both information detection and $\mathrm{FH}$ pattern acquisition. Following this initial blind $\mathrm{FH}$ pattern acquisition the MS receives the $\mathrm{FH}$ pattern code from the BS and may proceed to the information reception phase by exploiting the explicit knowledge of the $\mathrm{FH}$ pattern. However, the proposed blind-detection solutions can be applied by both the BS and MS.

\section{SFH/MC DS-CDMA SYSTEM}

\subsection{Transmitter Model}

The transmitter schematic along with the multiple access channel is shown in Fig. 1. There are $K$ users in the system and each subcarrier of each user is assigned a random spreading sequence. In the schematic $\mathrm{C}\left(Q, U_{k}\right)$ denotes a constant-weight code assigned to user $k$, which has $U_{k}$ number of binary ' 1 's, indicating the activated $\mathrm{FH}$ carriers. Hence the weight of $\mathrm{C}\left(Q, U_{k}\right)$ is $U_{k}$. This code is selected from the constant-weight code book of the schematic, which describes the FH patterns. There are $\left(\begin{array}{c}Q \\ U_{k}\end{array}\right)=Q ! / U_{k} !(Q-$ $\left.U_{k}\right)$ ! number of possible such weight- $U_{k}$ sequences and the constant-weight code $\mathrm{C}\left(Q, U_{k}\right)$ describes the $\mathrm{FH}$ patterns employed. Specifically, $\mathrm{C}\left(Q, U_{k}\right)$ determines the positions of the $U_{k}$ number of binary ' 1 's, which control the selection 


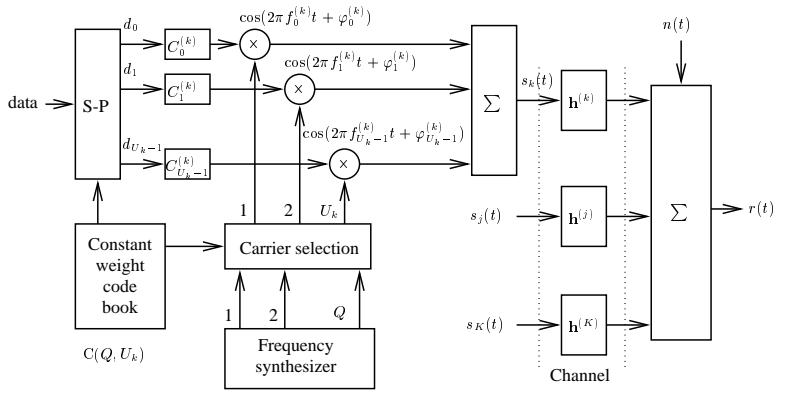

Figure 1: Transmitter and channel block diagram of the frequency-hopping multicarrier DS-CDMA systems.

of a set of $U_{k}$ number of active $\mathrm{FH}$ subcarrier frequencies from the $Q$ outputs of the frequency synthesizer.

The original bit stream having a bit duration of $T_{b}$ is first serial-to-parallel (S-P) converted, as seen in the schematic, yielding $U_{k}$ parallel streams. The assignment of bits to the activated subcarriers is controlled by the constantweight code $\mathrm{C}\left(Q, U_{k}\right)$. Depending on the specific aims of the system design, we can assign every $U_{k}$-th bit to the same subcarrier and in this parallel system the bit-duration is hence extended to $T=U_{k} T_{b}$. This assists in mitigating the inter-symbol-interference (ISI) in a high-bit rate transmission scheme. Alternatively, the proposed parallel system can be used to maintain $T=T_{b}$ in each subchannel, hence assisting in high-rate or variable-rate transmission upon employing a different number of subcarriers, as we will highlight during our further discourse. Theoretically, $Q$ number of different information rates can be generated by changing the weight of the code $\mathrm{C}\left(Q, U_{k}\right)$. As seen in Fig. 1, the direct-sequence (DS) spread, transmitted signal of the $k$ th user can be formulated as:

$$
s_{k}(t)=\sum_{u_{k}=0}^{U_{k}-1} \sqrt{2 P} d_{u_{k}}^{(k)}(t) c_{u_{k}}^{(k)}(t) \cos \left(2 \pi f_{u_{k}}^{(k)} t+\varphi_{u_{k}}^{(k)}\right),
$$

where $P$ denotes the transmitted power per carrier, while $U_{k}$ is the weight of the constant-weight $\mathrm{FH}$ code of the $k$ th user. Furthermore, $\left\{d_{u_{k}}^{(k)}(t)\right\},\left\{c_{u_{k}}^{(k)}(t)\right\},\left\{f_{u_{k}}^{(k)}\right\}$ and $\left\{\varphi_{u_{k}}^{(k)}\right\}$ denote the current data stream's waveforms, the DS spreading waveforms, the subcarrier frequency set and the phase angles introduced in the carrier modulation process, respectively. Let $T_{c}$ be the chip duration of the DS spreading waveforms and $N=T_{b} / T_{c}$. Then the processing gain of $N_{P}=T / T_{c}$ equals to $U_{k} N$ or $N$, depending on the choice of the bit duration, as discussed previously. Furthermore, we assume that the $\mathrm{FH}$ interval is $T_{h}$, and that the number of data bits, $N_{b}=T_{h} / T$, transmitted per hop is a positive integer, which is strictly larger than 1, implying slow FH.

\subsection{Nakagami Channel Model}

We considered a frequency-selective multipath channel [3], whose complex low-pass impulse response for subcarrier $u_{k}$ of user $k$ is given by:

$$
h_{u_{k}}^{(k)}(t)=\sum_{l_{p}=0}^{L_{p}-1} \alpha_{u_{k}, l_{p}}^{(k)} e^{-j \phi_{u_{k}, l_{p}}^{(k)}} \delta\left(t-l_{p} T_{c}\right),
$$

where $l_{p} T_{c}$ is the relative delay of the $l_{p}$-th path of user $k$ with respect to the main path, the phases $\left\{\phi_{u_{k}, l_{p}}^{(k)}\right\}$ are independent identically distributed (iid) random variables uniformly distributed in the interval $(0,2 \pi)$, whilst the $L_{p}$ tap weights $\left\{\alpha_{u_{k}, l_{p}}^{(k)}\right\}$ are independent Nakagami- $m$ random variables with a pdf of [4]:

$$
\begin{aligned}
& p\left(\alpha_{u_{k}, l_{p}}^{(k)}\right)=M\left(\alpha_{u_{k}, l_{p}}^{(k)}, m, \Omega_{u_{k}, l_{p}}^{(k)}\right), \\
& M(R, m, \Omega)=\frac{2 m^{m} R^{2 m-1}}{\Gamma(m) \Omega^{m}} e^{(-m / \Omega) R^{2}},
\end{aligned}
$$

where $\Gamma(\cdot)$ represents the gamma function, and $m$ is the Nakagami- $m$ fading parameter, which is defined as $m=$ $E^{2}\left[\left(\alpha_{u_{k}, l_{p}}^{(k)}\right)^{2}\right] / \operatorname{Var}\left[\left(\alpha_{u_{k}, l_{p}}^{(k)}\right)^{2}\right]$. The parameter $\Omega_{u_{k}, l_{p}}^{(k)}$ is the second moment of $\alpha_{u_{k}, l_{p}}^{(k)}$, i.e $\Omega_{u_{k}, l_{p}}^{(k)}=E\left[\left(\alpha_{u_{k}, l_{p}}^{(k)}\right)^{2}\right]$. We assume a negative exponentially decaying multipath intensity profile (MIP) distribution given by:

$$
\Omega_{u_{k}, l_{p}}^{(k)}=\Omega_{u_{k}, 0}^{(k)} e^{-\eta l_{p}}, \quad \eta \geq 0 .
$$

where $\Omega_{u_{k}, 0}^{(k)}$ is the average signal strength corresponding to the first resolvable path and $\eta$ is the rate of average power decay.

\subsection{Receiver Model}

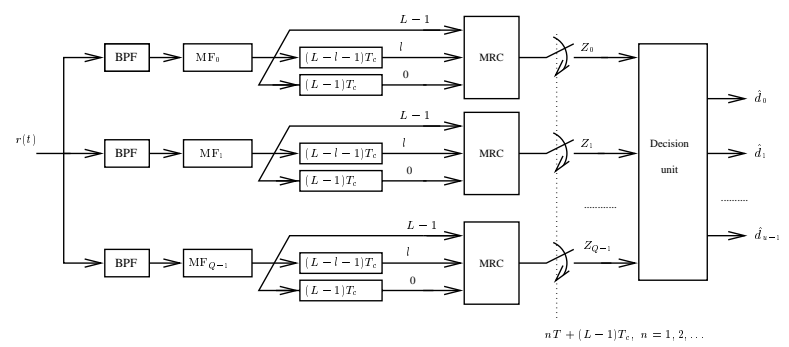

Figure 2: Receiver block diagram of the frequency-hopping multicarrier DS-CDMA systems.

We assume that the first user is the user-of-interest and consider the conventional matched filter based RAKE receiver with MRC of diversity order of $L$, as shown in Fig. 2 . In contrast to the transmitter side, where only $U_{k}$ out of $Q$ subcarriers are transmitted by the user $k$, at the receiver all $Q$ subcarriers are always tentatively demodulated. The information bits transmitted over the different subcarriers are blind soft-detected jointly using the MLD approach. Consequently, from the point of view of the receiver, each subcarrier can be viewed as an on-off type signalling scheme. When a subcarrier is actively used for signalling and hence it is in the on-state, the MRC output samples give +1 or -1 information, otherwise, while passive and hence in the off-state, the MRC stage outputs noise.

\section{BLIND SOFT-DETECTION OF SFH/MC DS-CDMA SIGNALS}

\subsection{Statistics of the MRC outputs}

The $Q$ number of matched filters in Fig. 2 are matched to the reference user's CDMA codes used for spreading in the 
sub-bands associated with the $Q$ number of subcarriers, and are assumed to have achieved time synchronization with the corresponding initial path of the subcarriers of the reference signal. If we assume that perfect estimates of the channel tap weights are available, then after appropriately delaying the individual matched filter outputs, in order to synchronize the $L$ number of path signals used by the RAKE combiner, the $q$ th MRC output sampled at $t=n T+(L-1) T_{c}$ in order to detect the $n$th symbol can be expressed as:

$$
Z_{q}[n]=D_{q}[n]+I_{q}[n] .
$$

Assuming that $Z_{q}[n]$ is an independent Gaussian distributed variable, ie invoking the Gaussian approximation for the multipath and multiple-access interference, then the mean value of $Z_{q}[n]$ can be expressed as:

$$
D_{q}[n]=\sqrt{\frac{P}{2}} T d_{q}(n) \sum_{l=0}^{L-1} \alpha_{q, l}^{2},
$$

where $d_{q}(n)$ is the $n$th bit transmitted on subcarrier $q$ by the reference user and $d_{q}(n) \in\{+1,-1,0\}$ with ' 0 ' representing the off-state. For simplicity, we assume that there exists no spectral overlap between the spectral main-lobes of two adjacent subcarriers [5], and interference is inflicted only, when an interfering user activates the same subcarrier, as the reference user, encountering a so-called hit [6]. Consequently, assuming that there exists $K_{h},\left(0 \leq K_{h} \leq K-1\right)$ number of interfering signals, all of which activate the $q$-th subcarrier during the $n$th symbol's transmission of the reference signal, the variance of $Z_{q}[n]$ can be expressed as:

$$
\begin{aligned}
\sigma^{2}=\frac{P T^{2}}{2}\left[\frac{q\left(L_{p}, \eta\right)-1}{2 N_{p}}\right. & +\frac{K_{h} q\left(L_{p}, \eta\right)}{3 N_{p}} \\
& \left.+\left(\frac{2 \Omega_{0} E_{b}}{N_{0}}\right)^{-1}\right] \Omega_{0} \sum_{l=0}^{L-1} \alpha_{q, l}^{2},
\end{aligned}
$$

where $E_{b}=P T$ is the average transmitted energy-per-bit and $q\left(L_{p}, \eta\right)=\left(1-e^{-\eta L_{p}}\right) /\left(1-e^{-\eta}\right)$, if $\eta \neq 0$, otherwise, $q\left(L_{p}, \eta\right)=L_{p}$, if $\eta=0$.

\subsection{Symbol-by-Symbol Maximum Likelihood De- tection}

Assuming that the receiver is aware of the weight of the transmitted constant-weight code, but not of the positions of the binary ' 1 's, the receiver has to detect not only the positions of the ' 1 's, which indicate the subcarriers used, but also the antipodal binary modulated information conveyed by the activated subcarriers. Let us express the $n$th input data in the vectorial form of $\mathbf{D}_{\mathbf{i}}=\left\{d_{i, 0}, d_{i, 1}, \ldots, d_{i, Q-1}\right\}$, where $0 \leq i \leq M-1$ represents the constant-weight code set of weight $U$ and $M$ is the number of weight- $U$ codes included in this set. Then a set of $Q$ MRC samples $\mathbf{Z}=$ $\left\{Z_{1}, Z_{2}, \ldots, Z_{Q-1}\right\}$ in Fig. 2 - which we refer to here as a received symbol or vector - are input to the 'decision unit' invoking a MLD, which is based on the computation of the probabilities defined as:

$$
p\left(\mathbf{Z} \mid \mathbf{D}_{\mathbf{i}}\right)=\frac{1}{\left(2 \pi \sigma^{2}\right)^{Q / 2}}
$$

$$
\begin{array}{r}
\cdot \exp \left(-\frac{\sum_{q=0}^{Q-1}\left(Z_{q}-\sqrt{\frac{P}{2}} T d_{i, q} \sum_{l=1}^{L-1} \alpha_{l}^{2}\right)^{2}}{2 \sigma^{2}}\right) \\
i=0,1, \ldots, M-1,
\end{array}
$$

where we assume that the subcarriers received over the same multipath ray undergo the same fading attenuation. The decision criterion is based on selecting the signal corresponding to the maximum of the set of probabilities $\left\{p\left(\mathbf{Z} \mid \mathbf{D}_{\mathbf{i}}\right)\right\}$.

The complexity of the MLD for a received symbol or vector is determined by both the length and the weight of the constant-weight code invoked. The average detection complexity order $(\mathrm{O})$ can be expressed as $O\left(3^{Q} / Q\right)$. Apparently, this complexity is excessive, rendering the associated detection complexity impractically high, when evaluating Eq.(8) for all possible code words, if the value of $Q$ is high. Hence we impose some limitations on the minimum distance of the codes in the constant-weight code-book representing the $\mathrm{FH}$ patterns, in order to simplify the detection. This reduces the size of the set.

Let $\mathrm{C}(Q, 2 v, U)$ represent a constant-weight code set having a code length of $Q$ and weight of $U$, as discussed previously. Furthermore, let the minimum distance between any pair of codes from $\mathrm{C}(Q, 2 v, U)$ be $2 v$, where $v$ is an integer larger than zero. Then, this code constitutes a specific, reduced-size subset of the constant-weight code $\mathrm{C}(Q, U)$, where the number of codewords was $M=\left(\begin{array}{l}Q \\ U\end{array}\right)$. By contrast, let $\mathrm{A}(Q, 2 v, U)$ represent the number of codewords of the constant-weight code $\mathrm{C}(Q, 2 v, U)$. Then, if the FH patterns are determined now by all the $\mathrm{A}(Q, 2 v, U)$ codewords, the detection complexity will be reduced from $O\left(3^{Q} / Q\right)$ to $O\left(\mathrm{~A}(Q, 2 v, U) 2^{U}\right)$.

\section{PERFORMANCE OF THE BLIND SOFT-DETECTION}

As shown for example in Fig. 3, for the SFH/MC DS-CDMA system using a constant-weight code $\mathrm{C}(Q, 2 v, U)$ in order to activate the subcarriers and using a receiver without the explicit knowledge of the FH pattern - except for knowing the number of the active subcarriers - we have to consider those codes, which have identical ' 1 ' positions in the FH code, corresponding to identical activated subcarriers. However, potentially different data symbols are conveyed on these active subcarriers. We refer to these as intra-set codes or intra-codes, for short. Explicitly, the above intra-codes are derived from a given constant-weight code and activate the same subcarriers. By contrast, the so-called inter-codes are derived from different constant-weight codes having the same number of ' 1 ' $\mathrm{FH}$ code positions, but activate different subcarriers.

Consequently, in our proposed blind soft-detection scheme two different types of code decision errors exist, the intracode errors and the inter-code errors, the probability of which is denoted by $P_{\text {intra }}(\cdot)$ and $P_{\text {inter }}(\cdot)$, respectively. The intra-code errors do not result in opting for a code other than the transmitter's code and hence do not inflict a constant-weight code decision error, they simply result in some bits being demodulated erroneously. By contrast, the inter-code errors may lead not only to erroneous bit decisions, but also to opting for another constant-weight code and hence to potentially more severe decision errors. 


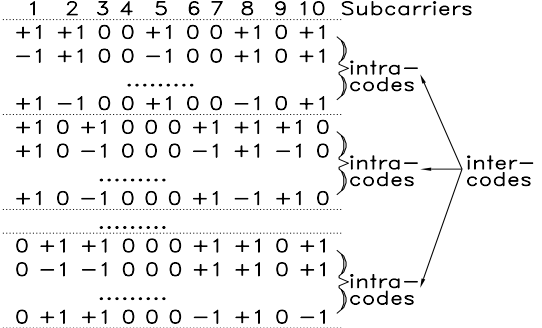

Figure 3: Example of intra-codes and inter-codes of a constant-weight code $\mathrm{C}(10,4,5)$ for an $\mathrm{SFH} / \mathrm{MC} \mathrm{DS}-$ CDMA system using 10 subcarriers.

It can be shown that the average probabilities of intracode error and inter-code error for a given number of hits, $K_{h}$, can be approximated as:

$$
\begin{aligned}
& P_{\text {intra }}\left(K_{h}, \gamma_{s}\right) \approx(U-1) P\left(K_{h}, \gamma_{s}\right), \\
& P_{\text {inter }}\left(K_{h}, \gamma_{s}\right) \approx 2^{v}[A(Q, 2 v, U)-1] P\left(K_{h}, \frac{v \gamma_{s}}{2}\right),
\end{aligned}
$$

where in Eq.(9) and Eq.(10) we have:

$$
\begin{gathered}
P\left(K_{h}, \gamma\right)=\sqrt{\frac{\gamma}{1+\gamma} \frac{(1+\gamma)^{-m_{s}} \Gamma\left(m_{s}+\frac{1}{2}\right)}{2 \sqrt{\pi} \Gamma\left(m_{s}+1\right)}} \\
{ }_{2} F_{1}\left(1, m_{s}+\frac{1}{2} ; m_{s}+1 ; \frac{1}{1+\gamma}\right), \\
\gamma=\frac{\bar{\gamma} \Omega_{s}}{2 m_{s}}=\frac{\bar{\gamma} q(L, 2 \eta)}{2 m q(L, \eta)}, \\
\bar{\gamma}=\left[\frac{q\left(L_{p}, \eta\right)-1}{2 N_{p}}+\frac{K_{h} q\left(L_{p}, \eta\right)}{3 N_{p}}+\left(\frac{2 \Omega_{0} E_{b}}{N_{0}}\right)^{-1}\right]^{-1},
\end{gathered}
$$

and $\Gamma(\cdot)$ is the Gamma function defined as $\Gamma(z)=\int_{0}^{\infty} e^{-t} t^{z-1} d t$ $z>0,{ }_{2} F_{1}(a, b, ; c ; z)$ is the hypergeometric function defined as [4]:

$$
{ }_{2} F_{1}(a, b, ; c ; z)=\sum_{k=0}^{\infty} \frac{(a)_{k}(b)_{k} z^{k}}{(c)_{k} k !}
$$

and $(a)_{k}=a(a+1) \cdots(a+k-1),(a)_{0}=1$.

The average bit error probability for a SFH based system can be expressed as [6]:

$$
P_{b}=\sum_{K_{h}=0}^{K-1}\left(\begin{array}{c}
K-1 \\
K_{h}
\end{array}\right) P_{h}^{K_{h}}\left(1-P_{h}\right)^{K-1-K_{h}} \bar{P}\left(K_{h}, \gamma_{s}\right)
$$

where $0 \leq K_{h} \leq K-1$ and $P_{h}$ is the probability of a hit from an interfering signal, which can be expressed as:

$$
P_{h}=\frac{\left(\begin{array}{c}
Q-1 \\
U_{k}-1
\end{array}\right)}{\left(\begin{array}{c}
Q \\
U_{k}
\end{array}\right)}=\frac{U_{k}}{Q}
$$

if we assume that the $\mathrm{FH}$ pattern of the $k$ th interfering user is determined randomly by a constant-weight code chosen from the set of $\left(\begin{array}{c}Q \\ U_{k}\end{array}\right)$ codes.

The probability of $\bar{P}\left(K_{h}, \gamma_{s}\right)$ in Eq.(14) denotes the conditional bit error probability, given that $K_{h}$ hits occurred from the other $K-1$ interfering users, which - according to Eq.(9) and Eq.(10) and upon omitting the detailed derivations - can be expressed as:

$$
\begin{aligned}
\bar{P}\left(K_{h}, \gamma_{s}\right)= & \frac{U-1}{U} P\left(K_{h}, \gamma_{s}\right) \\
& +\frac{2^{v}[A(Q, 2 v, U)-1]}{2} P\left(K_{h}, \frac{v \gamma_{s}}{2}\right) .
\end{aligned}
$$

\section{NUMERICAL RESULTS}

In this section, the average BER performance is evaluated as a function of the average signal-to-noise ratio (SNR) per bit, which is obtained by computing

$$
\bar{\gamma}_{b}=\frac{\left(1-e^{-L_{p} \eta}\right)}{1-e^{-\eta}} \cdot\left(\frac{\Omega_{0} E_{b}}{N_{0}}\right)=q\left(L_{p}, \eta\right)\left(\frac{\Omega_{0} E_{b}}{N_{0}}\right),
$$

for all systems described above.

Fig.4 shows the BER performance of the proposed SFH /MC DS-CDMA system with respect to the multipath fading parameters $m=0.5,1,2,5,10,50$. The range of other parameters used is explicitly stated in the corresponding figures. The Nakagami parameter $m$ represents the different fading environments, ranging from the worst-case onesided Gaussian fading to Rayleigh, and finally to the most favourable non-fading case. The parameters used are shown in the figure. As expected, for a given SNR per bit, the BER decreases upon increasing the value of $m$, which implies that the channel fading becomes less severe. The system performance is critically affected by the parameter $m$, ie by the communication environment encountered.

In Fig. 5 we evaluated the intra-code word error rate (WER) according to Eq.(9) and the inter-code WER from Eq.(10), as well as their sum for our SFH/MC DS-CDMA system using blind soft-detection. From the results we concluded that the total WER was typically dominated by one of its contributing factors. Namely, for very low SNR per bit values it was dominated by the WER of the inter-code , decisions, while for moderate to high SNRs per bit, by the WER of the intra-code errors. Since the inter-code WER of Eq.(10) is a function of $v$, hence, from Eq.(9) and Eq.(10) we infer that, if $v>2$, the total WER will be dominated by Eq.(9), provided that the SNR per bit is sufficiently high. This property suggests that for $v>2$ error-control techniques can be introduced, in order to correct the intracode errors by increasing the minimum distance between the intra-codes, and hence to decrease the intra-code WER of Eq.(9), and consequently to decrease the total WER of the blind soft-detection scheme.

Fig. 6 compared the performance of blind soft-detection to that of hard-detection, which benefitted from the explicit knowledge of the FH patterns employed. Different Nakagami fading parameters, namely $m=1,2,10$ were used, where the BER of hard-detection was introduced as a benchmarker. The results show that for a sufficiently high SNR per bit and a sufficiently good channel state, the BER performance of blind soft-detection was superior to that of the hard-detection. As we discussed in the context of Fig.5, if error-control is introduced for intra-code error-correction, then an improved BER performance can be achieved by blind soft-detection.

In Fig. 7 a multi-rate communication system was characterised using a constant processing gain of $N_{p}=N=256$. 
Let the rate of each transmitted subcarrier be $R_{b}$. Then the practical rates supported by the system might be $R_{b}$, $2 R_{b}, 4 R_{b}, 8 R_{b}, 16 R_{b}$, or $32 R_{b}$, since the constant-weight codes $\mathrm{C}(32,2,1), \ldots, \mathrm{C}(32,32)$ as shown in the figure were assumed. Due to the high minimum distance of the codes $\mathrm{C}(32,8,4), \mathrm{C}(32,14,8), \mathrm{C}(32,16,16)$, and $\mathrm{C}(32,32)$, we observe that even though the systems transmit at different rates, a more or less similar BER performance can be maintained, when the channel quality is sufficiently high - assuming appropriate constant-weight codes. By contrast, the BER performance of the system using the constant-weight codes of $\mathrm{C}(32,2,1)$ and $\mathrm{C}(32,4,2)$ was inferior with respect to the others', due to their relatively low minimum distance.

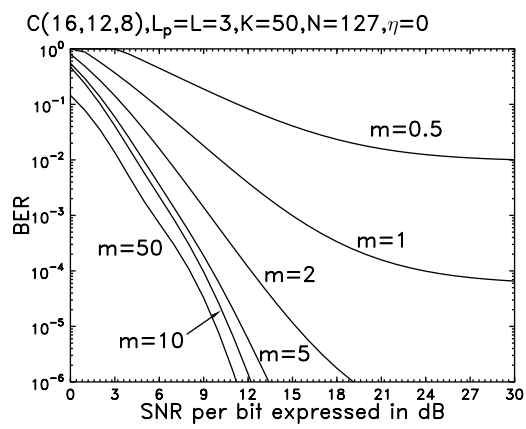

Figure 4: BER versus SNR per bit performance upon varying the fading parameter $m$ in the range of $\frac{1}{2}$ to 50 employing the constant-weight code $\mathrm{C}(16,12,8), L_{p}=3$ resolvable paths, diversity order $L=3, K=50$ users, bit-duration to chip-duration ratio $N=127$ and MIP decay factor $\eta=0$.

\section{REFERENCES}

[1] E. Dahlman, B. Gudmundson, M. Nilsson and J. Skold, "UMTS and IMT-2000 based on wideband CDMA," IEEE Commun. Mag., Vol. 36, No. 9, pp. 70-80, Sept. 1998.

[2] N. Nakagami, "The $m$-distribution, a general formula for intensity distribution of rapid fading," in Statistical Methods in Radio Wave Propagation, W. G. Hoffman, ed. Oxford, England:Pergamon, 1960.

[3] J. G. Proakis, Digital Communications, 3rd ed. New York: McGraw-Hill, 1995.

[4] T. Eng and L. B. Milstein, "Coherent DS-CDMA performance in Nakagami multipath fading," IEEE Trans. on Commun., Vol. 43, No. 2/3/4, pp. 11341143, Feb./Mar./Apr. 1995.

[5] S. Kondo and L. B. Milstein, "Performance of multicarrier DS CDMA systems," IEEE Trans. on Commun., Vol. 44, No. 2, pp. 238-246, Feb. 1996.

[6] T. Vlachos and E. Geraniotis, "Performance study of hybrid spread-spectrum random-access communications," IEEE Trans. on Commun., Vol. 39, No. 6, pp.975-985, June 1991.

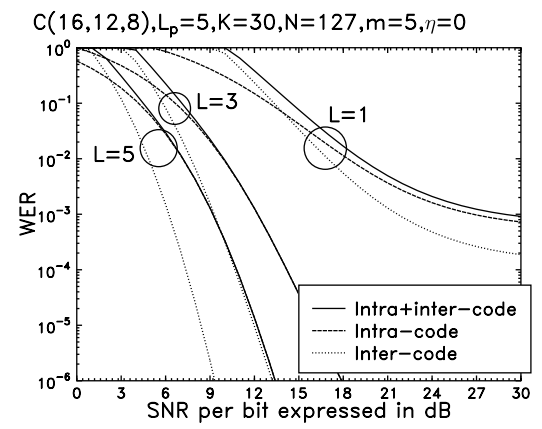

Figure 5: WER versus SNR per bit performance of blind soft-detection upon varying the diversity order $L$.

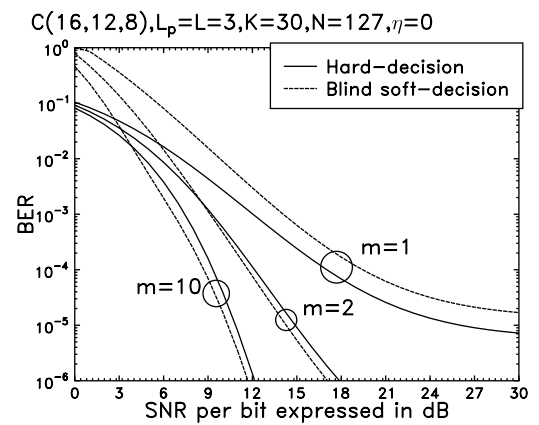

Figure 6: BER versus SNR per bit performance comparison between hard-detection and blind soft-detection upon varying the fading parameter $m$.

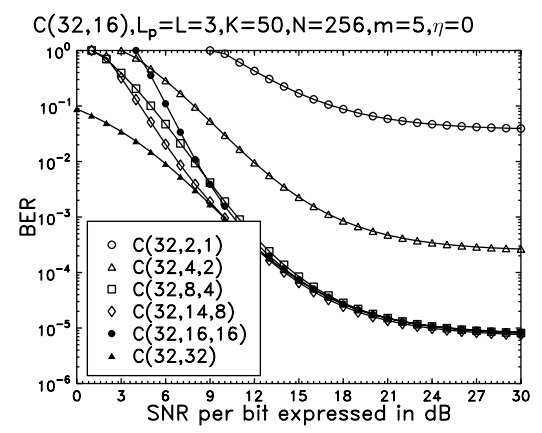

Figure 7: BER versus SNR per bit performance of blind soft-detection under the assumption of constant spreading gain for multi-rate based systems. 